\title{
Inequalities for the Polar Derivative of A Polynomial
}

\author{
M.S. Pukhta \\ Division of Agri. Statistics, Sher-e-Kashmir University of Agricultural Sciences and Technology of Kashmir, 191121, India \\ *Corresponding Author: mspukhta_67@yahoo.co.in
}

Copyright (C2013 Horizon Research Publishing All rights reserved.

Abstract If $P(z)=a_{n} z^{n}+\sum_{v=\mu}^{n} a_{n-v} z^{n-v}, 1 \leq \mu \leq n$ has all its zeros on $|z|=k, k \leq 1$, then it was recently proved by Dewan and Ahuja [3] that for every real or complex number $\alpha$ with $|\alpha| \leq k$.

$$
\max _{|z|=1}\left|D_{\alpha} P(z)\right| \leq \frac{n\left(|\alpha|+s_{\mu}\right) s_{\mu}}{k^{n-\mu+1}\left(1+s_{\mu}\right)} \max _{|z|=1}|P(z)|,
$$

where $s_{\mu}=\frac{n\left|a_{n}\right| k^{2 \mu}+\mu\left|a_{n-\mu}\right| k^{\mu-1}}{n\left|a_{n}\right| k^{\mu-1}+\mu\left|a_{n-\mu}\right|}$.

In this paper, we improve the above result and obtain new inequality for the polar derivative of a polynomial.

Keywords Polynomials, zeros, Maximum modulus, Polar derivative.

MSC (2010) 30A10; 30C10; 30C15

\section{Introduction and Statement of Results}

Let $P(z)$ be a polynomial of degree $n$ and $P^{\prime}(z)$ denotes its derivative. It was shown by Turan [8] that if $P(z)$ has all its zeros in $|z|=1$, then

$$
\max _{|z|=1}\left|P^{\prime}(z)\right| \geq \frac{n}{2} \max _{|z|=1}|P(z)| .
$$

As an extension of (1.1), Malik [9] proved that if $P(z)$ has all its zeros in $|z| \leq k, k \leq 1$, then

$$
\max _{|z|=1}\left|P^{\prime}(z)\right| \geq \frac{n}{1+k} \max _{|z|=1}|P(z)| \text {. }
$$

As a refinement of (1.2) it was shown by Govil [7] that if $P(z)$ has all its zeros in $|z| \leq k, k=1$, then

$$
\max _{|z|=1}\left|P^{\prime}(z)\right| \geq \frac{n}{1+k}\left\{\max _{|z|=1}|P(z)|+\frac{1}{k^{n-1}} \min _{|z|=k}|P(z)|\right\} \text {. }
$$

Aziz and Shah [1] generalized (1.3) in a different direction and proved that if $P(z)=a_{n} z^{n}+\sum_{v=\mu}^{n} a_{n-v} z^{n-v}$,
$1 \leq \mu \leq n$ is a polynomial of degree $n$ having all its zeros in $|z| \leq k, k \leq 1$, then

$\max _{|z|=1}\left|P^{\prime}(z)\right| \geq \frac{n}{1+k^{\mu}}\left\{\max _{|z|=1}|P(z)|+\frac{1}{k^{n-\mu}} \min _{|z|=k}|P(z)|\right\}$.

Let $\alpha$ be any complex number. If $P(z)$ is a polynomial of degree $n$, then the polar derivative of $P(z)$ with respect to the point $\alpha$, denoted by $D_{\alpha} P(z)$, is defined by

$$
D_{\alpha} P(z)=n P(z)+(\alpha-z) P^{\prime}(z)
$$

The polynomial $D_{\alpha} P(z)$ is of degree atmost $n-1$ and it generalize the ordinary derivative in this sense that

$$
\lim _{\alpha \rightarrow \infty}\left\{\frac{D_{\alpha} P(z)}{\alpha}\right\}=P^{\prime}(z) .
$$

Dewan, Singh and Lal [4] extended inequality (1.4) to the polar derivative of a polynomial and proved that if all the zeros of $P(z)=a_{n} z^{n}=\sum_{v=\mu}^{n} a_{n-v} z^{n-v}$, we have $1 \leq \mu \leq n$, lie in $|z|=k, k \leq 1$, then for every real or complex number a with $|\alpha| \geq k$

$$
\begin{aligned}
\max _{|z|=1}\left|D_{\alpha} P(z)\right| \geq & \frac{n\left(|\alpha|-k^{\mu}\right)}{1+k^{\mu}} \max _{|z|=1}|P(z)| \\
& \quad+\frac{n(|\alpha|+1)}{k^{n-\mu}\left(1+k^{\mu}\right)} \min _{|z|=k}|P(z)| .
\end{aligned}
$$

If we divide both sides of (1.5) by $|\alpha|$ and let $|\alpha| \rightarrow \infty$, we get inequality (1.4).

On the other hand, Dewan and Ahuja [3] recently proved for the class of polynomials having zeros on the boundary of a circle that

Theorem 1. If $P(z)=a_{n} z^{n}+\sum_{v=\mu}^{n} a_{n-v} z^{n-v}, 1 \leq \mu \leq n$, has all its zeros on $|z|=k, k \leq 1$, then for every real or complex number $\alpha$ with $|\alpha| \geq k$,

$$
\max _{|z|=1}\left|D_{\alpha} P(z)\right| \leq \frac{n\left(|\alpha|+s_{\mu}\right)}{k^{n-2 \mu+1}+k^{n-\mu+1}} \max _{|z|=1}|P(z)|
$$

where

$$
s_{\mu}=\frac{n\left|a_{n}\right| k^{2 \mu}+\mu\left|a_{n-\mu}\right| k^{\mu-1}}{n\left|a_{n}\right| k^{\mu-1}+\mu\left|a_{n-\mu}\right|}
$$


In this paper we shall prove the following result which is an improvement of Dewan and Ahuja [3]. The bound obtained by our Theorem gives a better bound than the bound of Theorem A.

Theorem 2. If $P(z)=a_{n} z^{n}+\sum_{v=\mu}^{n} a_{n-v} z^{n-v}, 1 \leq \mu \leq n$ is a polynomial of degree $n$ having all its zeros on $|z|=k$, $k \leq 1$, then for every real or complex number $\alpha$ with $|\alpha|=k$, we have

$$
\max _{|z|=1}\left|D_{\alpha} P(z)\right| \leq \frac{n\left(|\alpha|+s_{\mu}\right) s_{\mu}}{k^{n-\mu+1}\left(1+s_{\mu}\right)} \max _{|z|=1}|P(z)|,
$$

where

$$
s_{\mu}=\frac{n\left|a_{n}\right| k^{2 \mu}+\mu\left|a_{n-\mu}\right| k^{\mu-1}}{n\left|a_{n}\right| k^{\mu-1}+\mu\left|a_{n-\mu}\right|}
$$

To prove that the bound obtained in the above theorem is better than the bound obtained in Theorem A, we have show that

$$
\frac{s_{\mu}}{k^{n-\mu+1}\left(1+s_{\mu}\right)} \leq \frac{1}{k^{n-2 \mu+1}+k^{n-\mu+1}}
$$

which is equivalent to

$$
s_{\mu}\left(1+\frac{1}{k^{\mu}}\right) \leq 1+s_{\mu}
$$

which implies

$$
s_{\mu} \leq k^{\mu}
$$

or

$$
\frac{n\left|a_{n}\right| k^{2 \mu}+\mu\left|a_{n-\mu}\right| k^{\mu-1}}{n\left|a_{n}\right| k^{\mu-1}+\mu\left|a_{n-\mu}\right|} \leq k^{\mu}
$$

As $k \leq 1$, this implies

$$
\frac{\mu}{n}\left|\frac{a_{n-\mu}}{a_{n}}\right| \leq k^{\mu}
$$

which is always true (see Lemma 4).

Remark. If we divide both sides of (1.7) by $|\alpha|$ and let $|\alpha| \rightarrow \infty$, we get

$$
\max _{|z|=1}\left|P^{\prime}(z)\right| \leq \frac{n s_{\mu}}{k^{n-\mu+1}\left(1+s_{\mu}\right)} \max _{|z|=1}|P(z)|,
$$

where $s_{\mu}$ is defined by (1.8).

\section{Lemmas}

For the proof of theorem, we need the following lemmas. The Lemma 1 is due to Aziz and Shah [1].

Lemma 1. If $P(z)=a_{n} z^{n}+\sum_{v=\mu}^{n} a_{n-v} z^{n-v}, 1 \leq \mu \leq n$ is a polynomial of degree $n$ having all its zeros in $|z| \leq k$, $k \leq 1$, then on $|z|=1$

$$
\left|q^{\prime}(z)\right| \leq k^{\mu}\left|P^{\prime}(z)\right|
$$

Lemma 2. If $P(z)=a_{n} z^{n}+\sum_{v=\mu}^{n} a_{n-v} z^{n-v}, 1 \leq \mu \leq n$, is a polynomial of degree $n$ having all its zeros in $|z| \leq k$, $k \leq 1$, then for every real or complex number $\alpha$ with $|\alpha| \geq k^{\mu}$, we have

$$
\left|D_{\alpha} P(z)\right| \geq n\left(\frac{|\alpha|-k^{\mu}}{1+k^{\mu}}\right)|P(z)|, \quad \text { for }|z|=1 .
$$

Proof of Lemma 2. Since $q(z)=z^{n} P\left(\frac{1}{\bar{z}}\right)$, we have

$$
q^{\prime}(z)=n z^{n-1} \overline{P^{\prime}\left(\frac{1}{\bar{z}}\right)}-z^{n-2} \overline{P^{\prime}\left(\frac{1}{\bar{z}}\right)} .
$$

Therefore, for $z=e^{i \theta} ; 0 \leq \theta<2 \pi$ we have

$$
q^{\prime}\left(e^{i \theta}\right)=n e^{i(n-1) \theta} \overline{P\left(e^{i \theta}\right)}-e^{i(n-2) \theta} \overline{P^{\prime}\left(e^{i \theta}\right)},
$$

which gives for $|z|=1$,

$$
\begin{aligned}
\left|q^{\prime}(z)\right| & =\left|n P(z)-z P(z)-z P^{\prime}(z)\right| \\
& \geq n|P(z)|-\left|P^{\prime}(z)\right|,
\end{aligned}
$$

or

$$
\left|P^{\prime}(z)\right|+\left|q^{\prime}(z)\right| \geq n|P(z)|, \quad \text { for }|z|=1 .
$$

On combining (2.1) and (2.3), we obtain

$$
\begin{aligned}
\left(1+k^{\mu}\right) P^{\prime}(z) \mid & \geq\left|P^{\prime}(z)\right|+\left|q^{\prime}(z)\right| \\
& \geq n \mid P(z)
\end{aligned}
$$

or

$$
\left|P^{\prime}(z)\right| \geq\left(\frac{n}{1+k^{\mu}}\right)|P(z)|, \quad \text { for }|z|=1 .
$$

Now for every real or complex number $\alpha$, with $|\alpha| \geq k^{\mu}$, the polar derivative of $P(z)$ with respect to $\alpha$ is

$$
D_{\alpha} P(z)=n P(z)+(\alpha-z) P^{\prime}(z)
$$

This implies for $|z|=1$,

$$
\begin{aligned}
\left|D_{\alpha} P(z)\right| & \geq|\alpha|\left|P^{\prime}(z)\right|-\left|n P(z)-z P^{\prime}(z)\right| \\
& \geq|\alpha|\left|P^{\prime}(z)\right|-\left|q^{\prime}(z)\right| .
\end{aligned}
$$

Inequality (2.5) when combined with (2.1) of Lemma 1, gives (2.6)

$$
\left|D_{\alpha} P(z)\right| \geq\left(|\alpha|-\left|k^{\mu}\right|\right)\left|P^{\prime}(z)\right|, \quad \text { for }|z|=1 .
$$

The above inequality (2.6) in conjunction with (2.4) gives

$$
\left|D_{\alpha} P(z)\right| \geq n\left(\frac{|\alpha|-k^{\mu}}{1+k^{\mu}}\right)|P(z)|, \quad \text { for }|z|=1 .
$$

which proves the Lemma.

Lemma 3. If $P(z)=a_{n} z^{n}+\sum_{v=\mu}^{n} a_{n-v} z^{n-v}, 1 \leq \mu<n$, is a polynomial of degree $n$ having no zeros in $|z|<k$, $k \leq 1$, then for $|z|=1$,

$$
k^{n-\mu+1} \max _{|z|=1}\left|P^{\prime}(z)\right| \leq \max _{|z|=1}\left|q^{\prime}(z)\right| .
$$


The above lemma is due to Dewan and Hans [5].

Lemma 4. If $P(z)=a_{n} z^{n}+\sum_{v=u}^{n} a_{n-v} z^{n-v}, 1 \leq \mu \leq n$, is a polynomial of degree $n$ having all its zeros in $|z| \leq k$, $k \leq 1$ then for $|z|=1$,

$$
\left|q^{\prime}(z)\right| \leq s_{\mu}\left|P^{\prime}(z)\right|
$$

and

$$
\frac{\mu}{n}\left|\frac{a_{n-\mu}}{a_{n}}\right| \leq k^{\mu}
$$

where $s_{\mu}$ is defined by (1.8)).

The above lemma is due to Aziz and Rather [2].

Lemma 5. If $P(z)$ is a polynomial of degree $n$, then for $|z|=1$.

$$
\left|P^{\prime}(z)\right|+\left|q^{\prime}(z)\right| \leq n \max _{|z|=1}|P(z)|
$$

The above lemma is a special case of a result due to Govil and Rahman [6].

\section{Proof of Theorem}

Proof of Theorem. Let $z_{0}$ be a point on $|z|=1$ such that $\left|q^{\prime}\left(z_{0}\right)\right|=\max _{|z|=1}\left|q^{\prime}(z)\right|$, then by Lemma 5 , we get

$$
\left|P^{\prime}\left(z_{0}\right)\right|+\max _{|z|=1}\left|q^{\prime}(z)\right| \leq n \max _{|z|=1}|P(z)| .
$$

Combining the above inequality (3.1) with inequality (2.8) of Lemma 4, we have

$$
\frac{1}{s_{\mu}}\left|q^{\prime}\left(z_{0}\right)\right|+\max _{|z|=1}\left|q^{\prime}(z)\right| \leq n \max _{|z|=1}|P(z)|,
$$

which is equivalent to

$$
\left(\frac{1}{s_{\mu}}+1\right) \max _{|z|=1}\left|q^{\prime}(z)\right| \leq n \max _{|z|=1}|P(z)| .
$$

The above inequality (3.2) when combined with Lemma 3, gives

$$
k^{n-\mu+1}\left(\frac{1}{s_{\mu}}+1\right) \max _{|z|=1}\left|P^{\prime}(z)\right| \leq n \max _{|z|=1} \mid P(z) .
$$

which implies

$$
\max _{|z|=1}\left|P^{\prime}(z)\right| \leq \frac{n s_{\mu}}{k^{n-\mu+1}\left(1+s_{\mu}\right)} \max _{|z|=1} \mid P(z) .
$$

Now if $q(z)=z^{n} \overline{P\left(\frac{1}{\bar{z}}\right)}$, then as in the proof of Lemma 2, it easily follows that

$$
\left|q^{\prime}(z)\right|=n P(z)+(\alpha-z) P^{\prime}(z) .
$$

Also for every real or complex number $\alpha$ with $|\alpha| \geq k$, we have

$$
D_{\alpha} P(z)=n P(z)+(\alpha-z) P^{\prime}(z)
$$

This implies with the help of Lemma 4 that for $|z|=1$,

$$
\begin{aligned}
\left|D_{\alpha} P(z)\right| & \leq|\alpha|\left|P^{\prime}(z)\right|+\left|n P(z)-z P^{\prime}(z)\right| \\
& =|\alpha|\left|P^{\prime}(z)\right|+\left|q^{\prime}(z)\right| \\
& \leq\left(|\alpha|+s_{\mu}\right)\left|P^{\prime}(z)\right| .
\end{aligned}
$$

Inequality (3.4) in conjunction with inequality (3.3) gives

$$
\max _{|z|=1}\left|D_{\alpha} P(z)\right| \leq \frac{n\left(|\alpha|+s_{\mu}\right) s_{\mu}}{k^{n-\mu+1}\left(1+s_{\mu}\right)} \max _{|z|=1}|P(z)|,
$$

This completes the proof of main theorem.

\section{REFERENCES}

[1] A. Aziz and W.M. Shah, An integral mean estimate for polynomials, Indian J. Pure Appl. Math., Vol. 28 (1997), 1413-1419.

[2] A. Aziz and N.A. Rather, Some Zygmund type inequalities for polynomials, J. Math. Anal. Appl., Vol. 289 (2004), 14-29.

[3] K.K. Dewan and Arty Ahuja, On extremal properties for the polar derivative of polynomials, Anal. Theory Appl., Vol. 27 (2011), 150-157.

[4] K.K. Dewan, N. Singh and R. Lal, Inequalities for the polar derivative of a polynomial, Intl. J. Pure. Appl. Math., Vol. 33 (2006), 109-117.

[5] K.K. Dewan and S. Hans, On extremal properties for the derivative of polynomials, Math. Balkanica New Series, Vol. 23 (2009), 27-36.

[6] N.K. Govil and Q.I. Rahman, Functions of exponential type not vanishing in a half-plane and related polynomials, Trans. Amer. Math. Soc., Vol. 137 (1969), 501-517.

[7] N.K. Govil, Some inequalities for derivative of polynomials, J. Approx. Theory, Vol. 66 (1991), 29-35.

[8] P. Turan, Uber die ableitung von polynomen, Composition Math., Vol. 7 (1939). 89-95.

[9] M.A. Malik, On the derivative of a polynomial, J. London Math. Soc., Vol. 2 (1969), 57-60. 